\title{
Improving the communication of hand hygiene procedures: Controlled observation, redesign, and randomized group comparisons
}

\author{
Francis T. Durso PhD ${ }^{1}$ (1), Sweta Parmar MS ${ }^{1}$ (1), Ryan S. Heidish ${ }^{1}$, Skyler Tordoya Henckell BS ${ }^{1}$, Omer S. Oncul BS ${ }^{1}$ and \\ Jesse T. Jacob MD² (i) \\ ${ }^{1}$ School of Psychology, Georgia Institute of Technology, Atlanta, Georgia and ${ }^{2}$ Division of Infectious Diseases, Department of Medicine, Emory University, Atlanta, \\ Georgia
}

\begin{abstract}
Objective: To assess the clarity and efficacy of the World Health Organization (WHO) hand-rub diagram, develop a modified version, and compare the 2 diagrams.

Design: Randomized group design preceded by controlled observation and iterative product redesigns.

Setting: The Cognitive Ergonomics Lab in the School of Psychology at the Georgia Institute of Technology.

Participants: We included participants who were unfamiliar with the WHO hand-rub diagram (convenience sampling) to ensure that performance was based on the diagram and not, for example, on prior experience.

Methods: We iterated through the steps of a human factors design procedure: (1) Participants simulated hand hygiene using ultraviolet (UV) absorbent lotion and a hand-rub technique diagram (ie, WHO or a redesign). (2) Coverage, confusion judgments, and behavioral videos informed potentially improved diagrams. And (3) the redesigned diagrams were compared with the WHO version in a randomized group design. Coverage was assessed across 72 hand areas from multiple UV photographs.

Results: The WHO diagram led to multiple omissions in hand-surface coverage, including inadequate coverage by up to $75 \%$ of participants for the ulnar edge. The redesigns improved coverage significantly overall and often substantially.

Conclusions: Human factors modification to the WHO diagram reduced inadequate coverage for naïve users. Implementation of an improved diagram should help in the prevention of healthcare-associated infections.
\end{abstract}

(Received 6 June 2020; accepted 26 July 2020; electronically published 8 September 2020)

Hands of healthcare workers (HCWs) have been recognized as a common, critical intermediary in the transfer of pathogens from patient to patient ${ }^{1,2}$; thus, hand hygiene is regularly investigated and audited in hospitals. ${ }^{3-5}$ Hand hygiene research with HCWs has focused on compliance, ${ }^{2,6,7}$ training, ${ }^{8,9}$ amount and concentration of hand rub, ${ }^{10-13}$ and duration ${ }^{13,14}$ using direct observations, microbial counts on the hands, ultraviolet (UV) assessment of hands marked with a fluorescent dye, and fingertip cultures. ${ }^{2,15}$ The widely used World Health Organization (WHO) standard, virtually identical to the European standard (EN1500), has been studied using HCWs and has been compared to other diagrams (eg, Centers for Disease Control and Prevention [CDC] 3-step diagram) and to undirected "responsible application." ${ }^{\text {"16,17 }}$

\section{Author for correspondence: Frank Durso, E-mail: frank.durso@gatech.edu}

Cite this article: Durso FT, et al. (2021). Improving the communication of hand hygiene procedures: Controlled observation, redesign, and randomized group comparisons. Infection Control \& Hospital Epidemiology, 42: 194-202, https://doi.org/ $10.1017 /$ ice. 2020.407
Direct observation has found inadequate hand surface coverage in $90 \%$ of HCWs. ${ }^{18}$ A UV-marked hand-rub technique using the $\mathrm{WHO} / \mathrm{EN} 1500$ diagram has shown inadequate coverage of hands in $\sim 30 \%$ of HCWs, with the dorsal surface of the hand routinely poorly covered. ${ }^{16,19-21}$ Fingertips, potentially the most contaminated parts of the hand during clinical care, ${ }^{22}$ may be frequently missed during hand hygiene practice. ${ }^{16,20,21}$ All of these findings suggest that inadequate hand surface coverage even by HCWs can result from following standard hand-rub techniques.

Recently, the coronavirus disease 2019 (COVID-19) pandemic made apparent the importance of being able to communicate clearly the appropriate way to clean hands not only to HCWs but also to patients, ${ }^{23}$ families, and the general public. The WHO has listed hand hygiene among the most effective preventive measures for preventing the spread of severe acute respiratory coronavirus virus 2 (SARS-CoV-2) among the general population via both self-contamination and cross contamination from others. ${ }^{24}$ Indeed, many public service efforts during the outbreak 
Fig. 1. Examples of ultraviolet camera pictures. Dark black color represents sunscreen coverage and bright color represents uncovered areas.

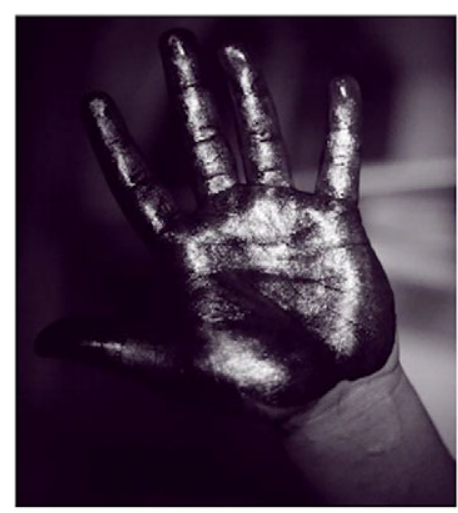

Virtually Covered Palmar Surface

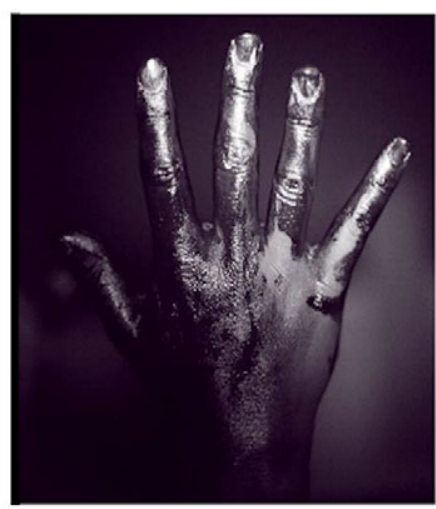

Noticeable Gaps on Dorsal Surface

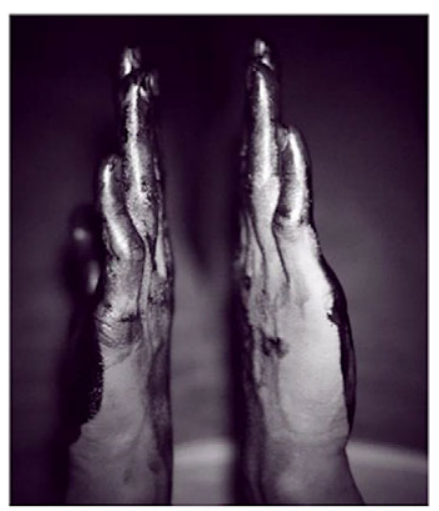

Virtually uncovered Ulnar Edge Body

have used the WHO diagram (ie, How To HandRub Poster.pdf) to create awareness for proper hand hygiene.

Although some research has been conducted in nonclinical communities on how and when hand hygiene is effective, ${ }^{25}$ including food service, ${ }^{26}$ collegiate, ${ }^{27,28}$ and other educational settings, ${ }^{29,30}$ the effectiveness of the diagrams responsible for communicating proper procedure to the general public has received little attention.

Human factors can play an important role in this public health crisis, ${ }^{31}$ as they have in the past. ${ }^{32-36}$ The goal of the current study was to evaluate and, if necessary, improve the WHO hand-rub diagram using human factors methods and principles to make hand hygiene easier, more natural, safer, and more efficient.

\section{Methods}

\section{Study design}

We used an iterative design approach. Following discussions with infectious disease researchers and practitioners and review of hand hygiene guidelines, we conducted several empirical studies. In phase 1, data were collected on the use of the WHO diagram to identify any problems and, if warranted, to inform its redesign. Phase 2 was a sequence of 2 studies comparing a redesigned diagram to the extant WHO diagram using equal allocation random assignment. Participants in phase 2 were randomly assigned in blocks of 2 participants to either the WHO diagram or a redesigned diagram, using a randomizer. ${ }^{37}$

\section{Setting and procedure}

In a standardized laboratory setting, participants were video recorded as they followed a diagram to simulate cleaning their hands using a gel with superior UV light absorption (Banana Boat 50+ SPF Ultra Sport sunscreen) as a surrogate alcohol-based hand-rub product. After completion, pictures of participants' hands were taken using a modified Canon EOS RP with a UV filter (Kolari Vision UV Bandpass) and UV flashlights. Coverage was assessed from pictures of several hand positions and angles. Following the simulation, participants ranked up to 4 confusing steps along with explanations of the confusing element.

A lead researcher prepared the randomized assignments and sequenced participant folders in service of a partial blind procedure used to keep awareness of the assigned group from the experimenters until they were required to pull the diagram from the folder.

Table 1. Participant Demographics

\begin{tabular}{lccc}
\hline Group & No. & Age, y & \% Male \\
\hline Phase 1: WHO & 30 & 20.6 & 50 \\
\hline Phase 2a: WHO & 30 & 18.9 & 63 \\
\hline Redesign & 30 & 19.0 & 50 \\
\hline Phase 2b: WHO & 29 & 19.7 & 41 \\
\hline Redesign & 29 & 19.6 & 52 \\
\hline
\end{tabular}

Note. WHO, World Health Organization.

Table 2. Correlations (Pearson $r$ ) Between Inadequate Coverage Across 72 Areas for Each of the WHO Groups Across 3 Phases

\begin{tabular}{lccc}
\hline Phase & $\begin{array}{c}\text { Pho 1: } \\
\text { WHO }\end{array}$ & $\begin{array}{c}\text { Phase 2a: WHO as } \\
\text { Control for 1st } \\
\text { Redesign }\end{array}$ & $\begin{array}{c}\text { Phase 2b: WHO as } \\
\text { Control for 2 } \\
\text { Redesign }\end{array}$ \\
\hline Phase 1 & .85 & .91 \\
\hline Phase 2a & $\ldots$ & $\ldots$ & .82 \\
\hline
\end{tabular}

Note. WHO, World Health Organization.

\section{Outcomes and analysis}

Two human factors researchers, blind to the participant's group, independently assessed each of 72 surface areas (36 per hand) on a 3-point scale: 0 , virtually uncovered; 1 , noticeable gaps; or 2 , virtually covered (Fig. 1). Reliability between the judges in each study was always $>80 \%$.

For the analysis of coverage, we used inadequate coverage scores (the combination of virtually uncovered and noticeable gaps). We computed the proportion of inadequately covered areas for each participant and submitted the data to the nonparametric MannWhitney $U$ test. Follow ups looked at the percentage of participants showing inadequate coverage for each hand area graphically.

\section{Participants}

To focus on the diagram as a causal factor in the coverage, rather than previous experience with clinical hand hygiene or with the WHO diagram, we recruited undergraduate volunteers. Participants were from the Georgia Institute of Technology in Atlanta, Georgia; they reported normal or corrected to normal vision. 


\begin{tabular}{|l|l|}
\hline \multicolumn{1}{c|}{ Rationale } \\
\hline $\begin{array}{l}\text { Coverage data: Not a factor. } \\
\text { Confusion data: Nota factor. } \\
\text { Video observations: Not a factor. } \\
\text { Iteration: The first redesign used the wHO } \\
\text { pictogram, but during the first iteration some users } \\
\text { found this step redundant and confusing, thinking } \\
\text { they had to take the sanitizer twice or put on both } \\
\text { hands separately }\end{array}$ \\
\hline $\begin{array}{l}\text { Coverage data: Not a factor. } \\
\text { Confusion data: Not a factor. } \\
\text { Video observations: Not a factor. }\end{array}$
\end{tabular}

Fig. 2. Design modifications and rationale.
In phase 1 (June 2019), 30 undergraduates participated in an exploratory study using the WHO diagram alone. In phase 2, the WHO diagram was compared with an initial (phase 2a) and then a final redesign (phase 2b). Power analyses to detect large effects suggested 30 participants per group. Phase 2a took place in the summer of 2019, with 60 undergraduate volunteers assigned randomly to use either the original WHO version or the modified version. We replaced 2 participants due to mechanical difficulties and 1 participant for ignoring the diagram completely. We again sought 60 participants for phase $2 \mathrm{~b}$ in the spring of 2020. However, data collection was halted on March 13,2020 , when the institute ceased on-campus instruction with the intent to move to remote learning because of the COVID-19 pandemic. Thus, we compared 29 students per group using the original WHO version or the second redesigned version.

\section{Redesign}

Beginning with the results of phase 1 and continuing through phase 2 , the redesign process itself began with hand areas that had a large percentage of participants with inadequate coverage. We then traced the area back to the steps in the diagram that were intended to cover that area. For each step, we looked at the videos to evaluate participant compliance and at the participants' confusion judgments.

\section{Results}

Gender and age information appear in Table 1. The participant sample in each of the 3 studies was typical of the Georgia Tech college population. The 3 correlations among the coverage scores of the WHO groups across studies were all high, confirming successful replication of the WHO exploratory study (Table 2).

\section{Phase 1: Assessment of the WHO diagram}

Figure 2 shows the WHO diagram steps (column 1), an overview of how the coverage, confusion, and video data informed the redesign process (column 2), and a final redesigned step (column 3). The design rationale relied on the synthesis of these 3 factors.

Coverage of the hand areas varied from exceptional (no participants inadequate) to several areas for which $>60 \%$ of participants covered inadequately. The poorest coverage was for the ulnar and radial edges of the hands and the dorsum. Because no step in the WHO diagram seemed to address the ulnar and radial edges of the hands, we added new steps $5 \mathrm{a}$ and $5 \mathrm{~b}$. Several other areas also suggested that there could be room for improvement.

Confusion also varied across the steps, with WHO steps 3, 5, and 7 most often considered confusing. Confusion judgments also alerted us to the particulars of the step that might be problematic (Fig. 2). The videos of hand rubbing often confirmed the participants' expressed confusion, but they also revealed issues that participants did not report. For example, in step 3, participants did not indicate confusion about rubbing the back of their hands, but they did not perform this action on the video. The videos also suggested that the natural flow of hand rubbing would benefit by moving step 7 in the modified diagram. ${ }^{14}$

The design modifications addressed the inadequately covered areas resulting from using the WHO diagram as well as the steps that were especially confusing or were incorrectly performed. The perspective and details of the original WHO depictions were modified to increase clarity. The design modifications were always made on the pictograms; none of the verbiage was changed. The final redesign is reproduced in Figure 3.

\section{Phase 2a: First redesign evaluation}

The redesigned diagram coverage (mean rank, 36.75) was significantly better than the coverage for participants in the WHO group 


\begin{tabular}{|c|c|c|}
\hline & $\begin{array}{l}\text { Similar coverage issues } \\
\text { manifested with the iteration. } \\
\text { Feedback and video helped us } \\
\text { modify the perspective and } \\
\text { hand orientation, resulting in } \\
\text { more curl in the fingers and } \\
\text { the addition of a curve in the }\end{array}$ & \\
\hline 4 & \multirow[t]{2}{*}{$\begin{array}{l}\text { Coverage data: Palm coverage was not a factor. } \\
\text { However, this step was also partly (along with Step } \\
\text { 3) responsible for webbing coverage, which was } \\
\text { somewhat inadequate. } \\
\text { Confusion data: A few users found curved arrows a } \\
\text { little confusing but making it straight does not } \\
\text { convey the motion. } \\
\text { Video observations: Not a factor. Because this step } \\
\text { showed no major errors in the video (and step } 3 \text { did) } \\
\text { we chose to modify Step } 3 \text {. }\end{array}$} & 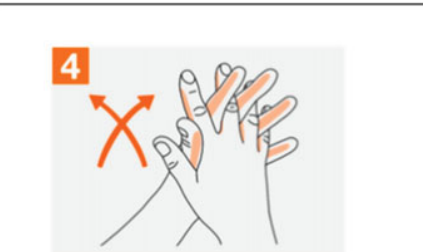 \\
\hline Palm to palm with fingers interlaced; & & Palm to palm with fingers interlaced; \\
\hline $\begin{array}{l}\text { Backs of fingers to opposing palms } \\
\text { with fingers interlocked; }\end{array}$ & $\begin{array}{l}\text { Coverage data: Nail segment coverage was } \\
\text { occasionally inadequate. } \\
\text { Confusion data: Curved arrows were confusing; } \\
\text { perspective was unclear. } \\
\text { Video observations: Rubbed nail tips to nail tips } \\
\text { (did not interlock fingers). } \\
\text { Iteration: }\end{array}$ & $\begin{array}{l}\text { Backs of fingers to opposing palms } \\
\text { with fingers interlocked; }\end{array}$ \\
\hline $\begin{array}{l}\text { Rotational rubbing of left thumb } \\
\text { clasped in right palm and vice versa; }\end{array}$ & $\begin{array}{l}\text { Coverage: Mediocre coverage of the thumb. } \\
\text { Confusion: A few found the arrows confusing. } \\
\text { Video: Not a factor. } \\
\text { Iteration: The first redesign used the WHO } \\
\text { pictogram, but during the first iteration some users } \\
\text { performed incorrect motion for this step by merely } \\
\text { holding the thumb. }\end{array}$ & $\begin{array}{l}\text { Rotational rubbing of left thumb } \\
\text { clasped in right palm and vice versa; }\end{array}$ \\
\hline \multirow[t]{2}{*}{$\begin{array}{l}\text { Rotational rubbing, backwards and } \\
\text { forwards with clasped fingers of right } \\
\text { hand in left palm and vice versa; }\end{array}$} & $\begin{array}{l}\text { Coverage data: Not a factor. } \\
\text { Confusion data: Users did not realize this step was } \\
\text { to cover fingertips. } \\
\text { Video observations: Users focused on finger } \\
\text { segments instead of tips. } \\
\text { Iteration: Our first iteration was thought to be } \\
\text { confusing both in hand } \\
\text { orientation (looked like } \\
\text { pinching the hand) and direction } \\
\text { of rubbing (arrows were placed } \\
\text { off-hand). A few users of the } \\
\text { iteration rubbed their fingers on } \\
\text { dorsum instead of palm or }\end{array}$ & $\begin{array}{l}\text { Rotational rubbing, backwards and } \\
\text { forwards with clasped fingers of right } \\
\text { hand in left palm and vice versa: }\end{array}$ \\
\hline & $\begin{array}{l}\text { Coverage data: Edges were some of the least } \\
\text { adequately covered areas for both hand edges. No } \\
\text { step in the original WHO diagram allowed for } \\
\text { coverage of the ulnar or radial edges of the hands. } \\
\text { Iteration: Our } \\
\text { first attempt } \\
\text { produced coverage } \\
\text { of edges that was } \\
\text { better than WHO } \\
\text { but was still } \\
\text { inconsistent. The iteration feedback allowed us to } \\
\text { tweak the smaller picture and extend the arrow } \\
\text { length. }\end{array}$ & 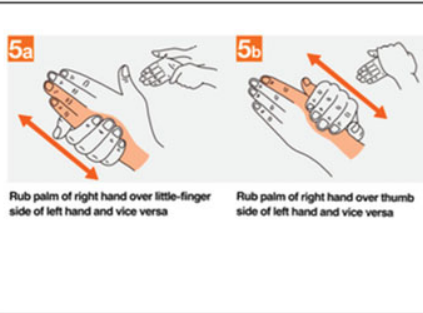 \\
\hline
\end{tabular}

Fig. 2. (Continued) length.

(mean rank, 24.25, Mann-Whitney $\mathrm{U}=262.5 ; P=.005 ;$ 2-tailed $\mathrm{r}=.358$ ). Thus, the median redesign participant covered more areas adequately than did the median WHO participant.

Looking at each area, coverage of most hand areas was improved compared to the WHO comparison group. Nevertheless, we felt that a second design iteration could improve coverage further. First, even though the first modification improved areas, coverage of some of those areas had noticeable deficits that we believed could be improved. For example, of the 72 areas, 20 areas showed inadequate coverage in $>15 \%$ of the participants. Detailed analysis of inadequate coverage along with confusion judgments regarding the steps and videos of the hand rubbing were again used to guide the development of the second design iteration.

In addition to specific problems identified in certain steps, participants did not always understand the area(s) that the steps were intended to cover. Human factors principles suggest that humans are often better if they can perceive a solution rather than think about one. Thus, in the second iteration, we shaded the areas that the step was intended to cover. Some steps required "ghosting" in which a part of the hand, which in reality would be occluded, was 


\section{How to Handrub?}

\section{RUB HANDS FOR HAND HYGIENE! WASH HANDS WHEN VISIBLY SOILED}

(1) Duration of the entire procedure: $20-30$ seconds

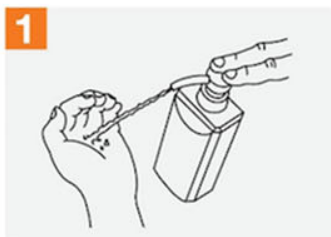

Apply a palmful of the product in a cupped hand, covering all surfaces;

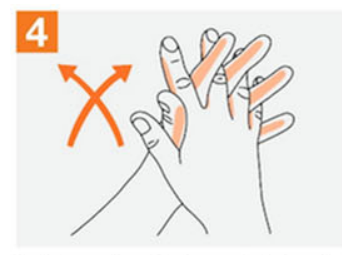

Palm to palm with fingers interlaced;
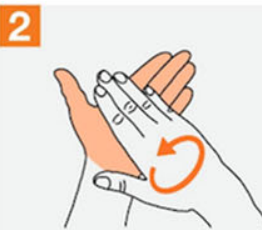

Rub hands palm to palm;

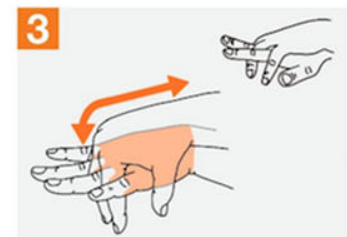

Right palm over left dorsum with interlaced fingers and vice versa;

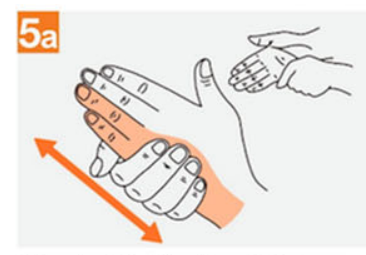

Rub palm of right hand over little-finger side of left hand and vice versa

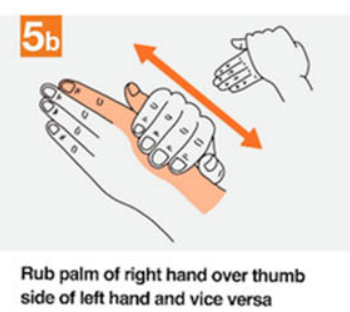

6

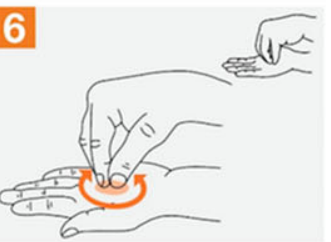

Rotational rubbing, backwards and forwards with clasped fingers of right hand in left palm and vice versa:

8

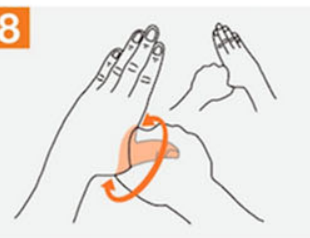

Rotational rubbing of left thumb clasped in right palm and vice versa;

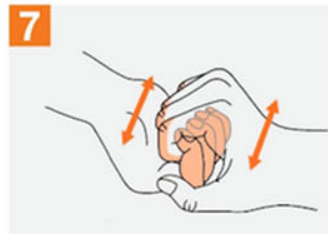

Backs of fingers to opposing palms with fingers interlocked;
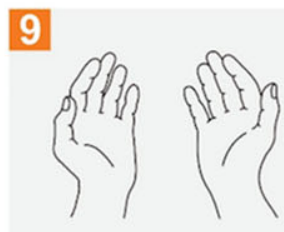

Once dry, your hands are safe.

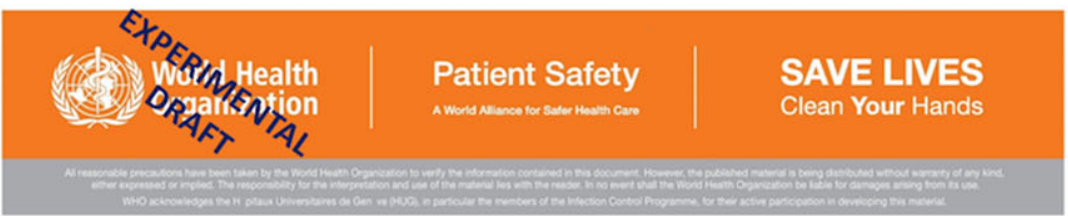

Fig. 3. Modification of the WHO diagram based on data from initial exploratory study and the first redesign. This version was compared with the original WHO diagram. Participants saw this diagram without the "experimental draft" overlay.

made visible (see eg, Fig. 3, step 8). Finally, given the success of the first redesign, we modified additional steps in an effort to reduce further the areas covered inadequately.

\section{Phase 2b: Final redesign evaluation}

Coverage for participants in the redesigned diagram group (mean rank, 33.5) again was significantly better than the coverage for participants in the WHO group (mean rank, 25.50, MannWhitney $\mathrm{U}=304.5 ; P=.035$; 1 -tailed, as expected, $r=.237$ ). Again, the median redesign participant covered fewer areas inadequately than did the median WHO participant. Moreover, $14 \%$ of the participants covered all areas adequately using the redesign, compared with $7 \%$ for the WHO diagram. The poorest performer covered $60 \%$ using the redesign, compared with $40 \%$ for the WHO group.

Figure 4 conveys an overview of the results of the final redesign by hand area. The abscissa of Figure 4 shows the WHO data, and the ordinate shows the second design iteration data. The line emerging from the origin represents equality. Data points below the line indicate superior performance for the redesigned diagram. Most hand areas were covered adequately by more participants using the redesign than using the WHO diagram. Several hand areas improved over 25 percentage points compared to the WHO version. In addition, the redesign yielded relatively few 


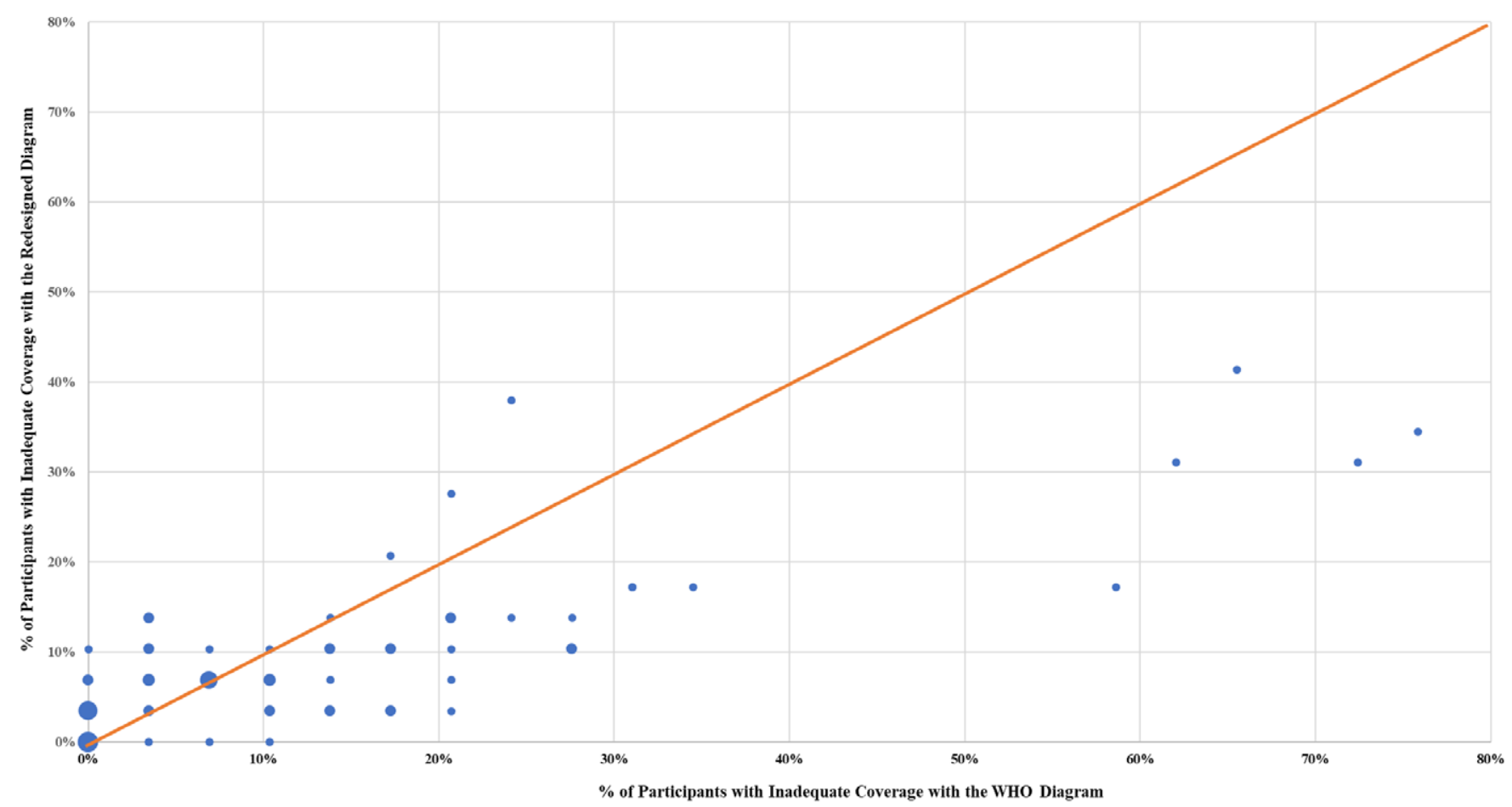

Fig. 4. Inadequate coverage for each of the 72 hand areas (\% of participants) for the Redesign (ordinate) and WHO (abscissa). Dot diameters reflect number of data points at those coordinates.

inadequate areas: In fact, 62 of the 72 hand areas were inadequately covered by $<15 \%$ of the participants, representing an improvement over the first iteration.

Our results do not imply poor performance with the original WHO diagram. In fact, for 30 of the 72 hand areas, all participants, or all but 1 , in the WHO group covered adequately (also true for 19 of these areas in the redesign). However, for most of the remaining hand areas, more participants using the redesigned diagram covered completely than those using the WHO diagram. Detailed heat maps (created using the app, Procreate) with the color saturation proportional to the percentage of participants with inadequate coverage data are presented in Figure 5.

\section{Ulnar edge of hand}

With the WHO diagram, the ulnar edge had the poorest coverage with over $70 \%$ of participants covering inadequately. The redesign's improvement was often substantial, as high as 42 percentage points.

\section{Dorsal surface of hand}

The dorsal hand coverage was inadequate using the WHO diagram, but it became gradually better as participants moved distally toward the fingertips. Improvement using the redesign was present in multiple comparisons and, again, was often substantial. As participants moved toward the nail tips, the redesign rarely improved over the WHO diagram, which was already performing fairly well.

\section{Radial edge of hand}

The radial lateral edge of the hand also showed problems with the WHO diagram. The bases of the thumbs were especially poor. The redesign again showed improvement in virtually all of the areas, with improvement as high as 42 percentage points.

\section{Interdigital web spaces}

Under the WHO diagram, on average, performance was fair and comparable for the 2 hands. This equivalence was not the case with the redesigned diagram. The redesign always outperformed the WHO diagram for the left hand, but virtually never did for the right hand. Further, only 3 hand areas were simultaneously poorer with the redesign and inadequate for $>15 \%$ of the participants (Fig. 4). All involved the interdigital web spaces of the right hand. The fact that the hands seem to differ in coverage may suggest a difference between dominant and nondominant hands in the performance of the modified step 3.

\section{Fingertips}

The fingertips were well covered in the WHO and redesign, as discussed earlier. Outperforming the WHO diagram at this level of performance was unlikely.

\section{Palmar (or volar) surface of hand}

Even more so than the fingertips, coverage of the palm by both diagrams was quite good.

\section{Discussion}

Potential gaps in the baseline WHO diagram were identified in several hand areas. By precise measurement of hand coverage along with procedures to identify difficulties and points of confusion and human factors methods, we were able to design and validate a redesigned diagram showing noticeable improvements in coverage in first time users.

We gained substantial insight for the redesign from the combination of video records, confusion, and coverage. Although coverage was our primary dependent variable driving the design decisions, both participant judgments of confusion and behavioral 

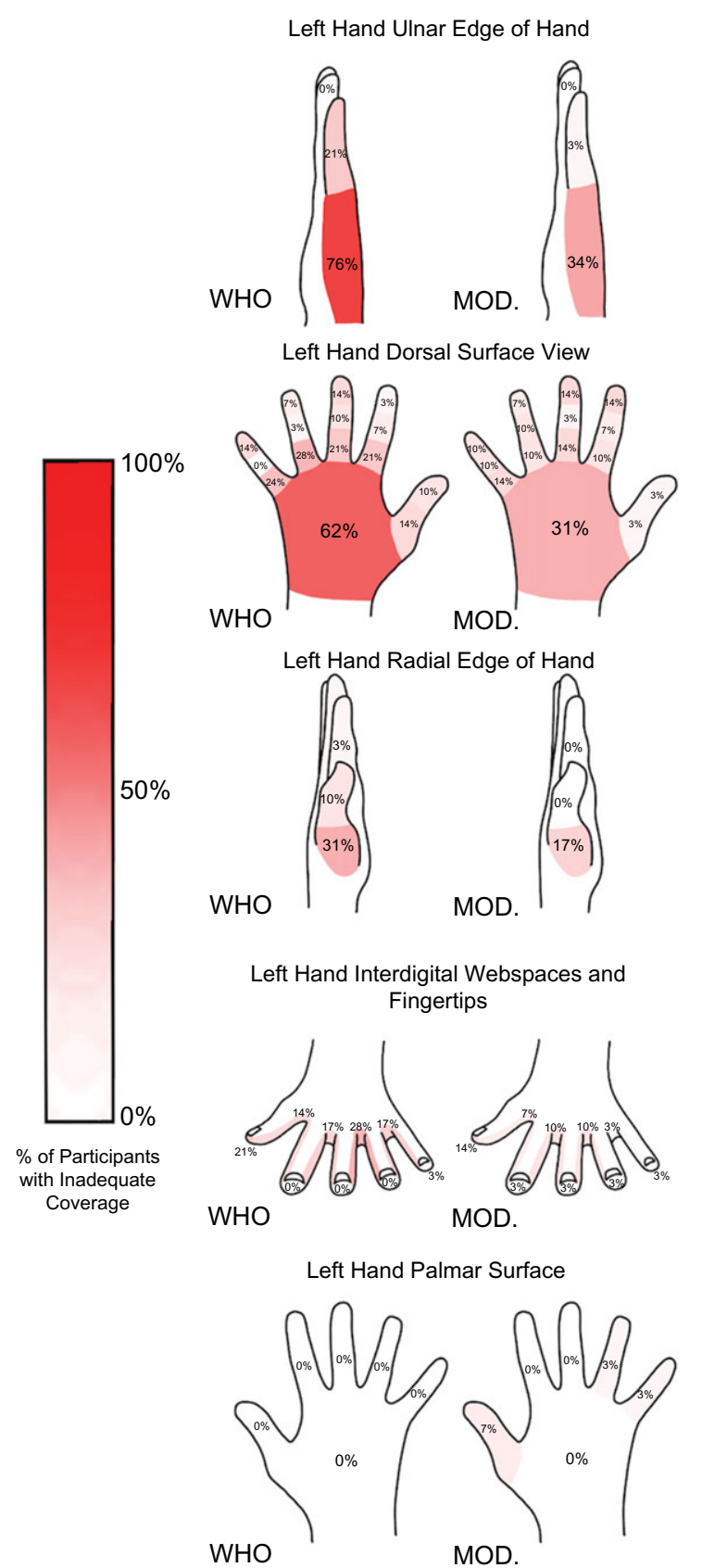

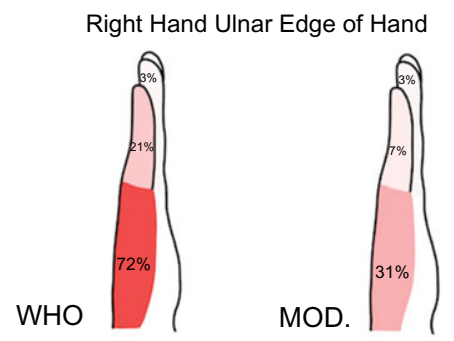

Right Hand Dorsal Surface View
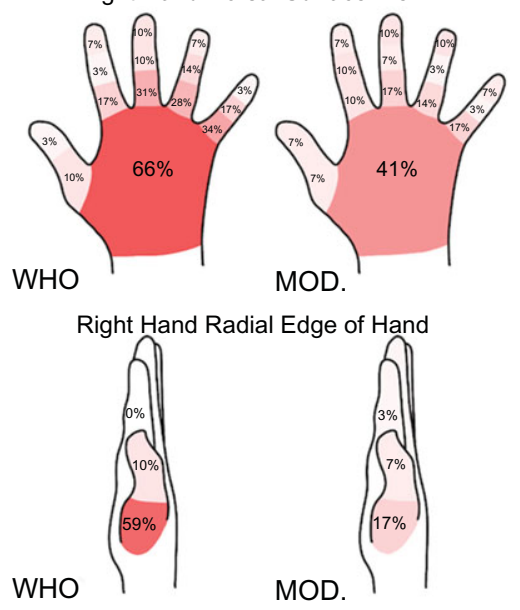

Right Hand Interdigital Webspaces and Fingertips

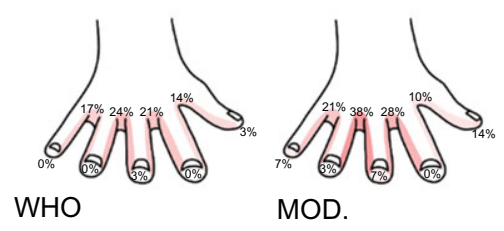

Right Hand Palmar Surface

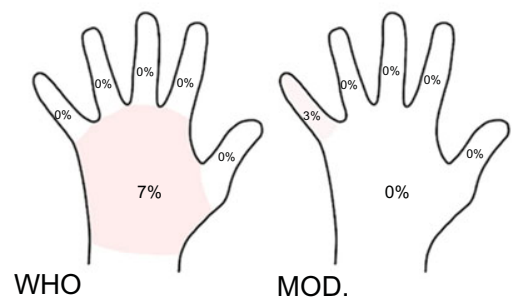

Fig. 5. Heat maps of inadequate coverage for both hands and both diagrams. Data represent the percentage of participants who had inadequate coverage (gaps or no coverage) of the indicated area. videos helped in identifying why coverage was bad or good for particular areas, steps, and participants. At times, poorly covered areas were accompanied by indications of confusion. In fact, the redesigned diagram reduced confusion for most of the steps; as one example, the confusion rating for step 8 reduced drastically when redesigned, showing the success of ghosting and highlighting. At other times, some coverage was exemplary even if participants indicated confusion. For example, several participants were confused about the fingertips step in both WHO and the redesigned diagrams, but coverage was quite good. On the other hand, participants might not express confusion, ${ }^{38}$ but the video indicated they continued the step in an incorrect way.

As might be expected with an iterative design procedure, several areas, although improved compared with the original, could be further improved. Surprisingly, although both redesign 1 and redesign 2 showed substantial improvements in the dorsal area of both hands and the ulnar edge of both hands, those 4 areas continue to have a large number of participants who sanitize inadequately (Fig. 4, 4 data points to the far right). We were also perplexed by the only moderate coverage of the web spaces, especially on the right hand and especially between the ring and middle finger, which may be a space physically difficult or awkward to cover. Although the inner web spaces rarely touch a patient or our faces, they might serve as sources of cross contamination, and deserve additional consideration. Notably, complete coverage of all hand surfaces may not be required to prevent contact transmission of infectious diseases, and this ideal might not be achieved with any number of redesign iterations. Adequate coverage of particular hand areas, a goal short of the ideal total coverage of all areas by all individuals, may be sufficient to prevent contact transmission.

Methodological limitations also exist of course. One is that results rest with the inherently subjective nature of human judges 
evaluating coverage; however, scores indicated high reliability between judges blinded to the diagram assignment, and our results were consistent across phases. The assessment and subsequent modifications of the WHO diagram were based on a relatively small and homogenous group of intelligent individuals presumably focused on and engaged with the task, all limiting generalizability. In addition, generalization should be limited to individuals who actually rely on the diagram to direct their behaviors and not on, for example, their prior experience. Finally, some of the larger benefits from the redesign were due to the addition of a step. Notably, the additional step may cost increased time for hand hygiene, which in turn could lead to lower compliance in field situations. Nevertheless, the redesigned diagram produced significant and sometimes substantial benefits in coverage.

The redesigned diagram can be deployed or it can be used to seed another design iteration. Regardless, the current study has identified clear gaps in the existing WHO diagram's ability to communicate to the general public and has offered procedures that can be used to investigate and ameliorate those deficits.

Acknowledgments. We thank Noushin Mannan, Taylor Helfrich, and Monica Montalvo for their help with the literature, preparation of materials, and data collection. We also thank Noushin Mannan for drawing the second redesigned diagram and the heat maps and Eric Stearman for initially detecting the gaps in the WHO data collection among healthcare workers as well as Joel Mumma for suggesting the use of sunscreen. Finally, thanks to Kate Bleckley for reviewing an earlier version of this work.

Financial support. No financial support was provided relevant to this article. JTJ was supported by the Prevention Epicenters Program of the Centers for Disease Control and Prevention (award U54CK000164).

Conflicts of interest. All authors report no conflicts of interest relevant to this article.

\section{References}

1. Boyce JM, Pittet D. Guideline for hand hygiene in health-care settings: recommendations of the Healthcare Infection Control Practices Advisory Committee and the HICPAC/SHEA/APIC/IDSA Hand Hygiene Task Force. Infect Control Hosp Epidemiol 2002;23:S3-S40.

2. Allegranzi B, Pittet D. Role of hand hygiene in healthcare-associated infection prevention. J Hosp Infect 2009;73:305-315.

3. Pessoa-Silva CL, Hugonnet S, Pfister R, et al. Reduction of health careassociated infection risk in neonates by successful hand hygiene promotion. Pediatrics 2007;120:e382-e390.

4. Pittet D, Allegranzi B, Sax H, et al. Evidence-based model for hand transmission during patient care and the role of improved practices. Lancet Infect Dis 2006;6:641-652.

5. Pittet D, Allegranzi B, Boyce J, World Alliance for Patient Safety First Global Patient Safety Challenge Core Group of Experts. The World Health Organization guidelines on hand hygiene in health care and their consensus recommendations. Infect Control Hosp Epidemiol 2009;30:611-622.

6. Chavali S, Menon V, Shukla U. Hand hygiene compliance among healthcare workers in an accredited tertiary-care hospital. Indian Soc Crit Care Med 2014;18:689-693.

7. Tschudin-Sutter S, Sepulcri D, Dangel M, Schuhmacher H, Widmer AF. Compliance with the World Health Organization hand hygiene technique: a prospective observational study. Infect Control Hosp Epidemiol 2015;36:482-483.

8. Sutter ST, Frei R, Dangel M, Widmer A. Effect of teaching recommended World Health Organization technique on the use of alcohol-based hand rub by medical students. Infect Control Hosp Epidemiol 2010;31:1194-1195.
9. Widmer AF, Conzelmann M, Tomic M, Frei R, Stranden AM. Introducing alcohol-based hand rub for hand hygiene: the critical need for training. Infect Control Hosp Epidemiol 2007;28:50-54.

10. Goroncy-Bermes P, Koburger T, Meyer B. Impact of the amount of hand rub applied in hygienic hand disinfection on the reduction of microbial counts on hands. J Hosp Infect 2010;74:212-218.

11. Kampf G, Ruselack S, Eggerstedt S, Nowak N, Bashir M. Less and lessinfluence of volume on hand coverage and bactericidal efficacy in hand disinfection. BMC Infect Dis 2013;13:472.

12. Macinga DR, Shumaker DJ, Werner H-P, et al. The relative influences of product volume, delivery format and alcohol concentration on dry-time and efficacy of alcohol-based hand rubs. BMC Infect Dis 2014;14:511.

13. Suchomel M, Kundi M, Pittet D, Weinlich M, Rotter ML. Testing of the World Health Organization recommended formulations in their application as hygienic hand rubs and proposals for increased efficacy. Am J Infect Control 2012;40:328-331.

14. Pires D, Bellissimo-Rodrigues F, Soule H, Gayet-Ageron A, Pittet D. Revisiting the WHO "how to handrub" hand hygiene technique: fingertips first? Infect Control Hosp Epidemiol 2017;38:230-233.

15. Kampf G, Loffler H, Gastmeier P. Hand hygiene for the prevention of nosocomial infections. Deutsches Ärzteblatt International 2009;106:649-655.

16. Kampf G, Reichel M, Feil Y, Eggerstedt S, Kaulfers P-M. Influence of rub-in technique on required application time and hand coverage in hygienic hand disinfection. BMC Infect Dis 2008;8:149.

17. Chow A, Arah OA, Chan S-P, et al. Alcohol handrubbing and chlorhexidine handwashing protocols for routine hospital practice: a randomized clinical trial of protocol efficacy and time effectiveness. Am J Infect Control 2012;40:800-805.

18. Park HY, Kim SK, Lim YJ, et al. Assessment of the appropriateness of hand surface coverage for health care workers according to World Health Organization hand hygiene guidelines. Am J Infect Control 2014;42:559-561.

19. Fichtner A, Haupt E, Karwath T, Wullenk K, Pöhlmann C, Jatzwauk L. A single standardized practical training for surgical scrubbing according to EN1500: effect quantification, value of the standardized method and comparison with clinical reference groups. GMS Zeitschrift für Medizinische Ausbildung 2013;30.

20. Skodova M, Gimeno-Benitez A, Martinez-Redondo E, et al. Hand hygiene technique quality evaluation in nursing and medicine students of two academic courses. Revista Latino-Am Enferm 2015;23:708-717.

21. Szilágyi L, Haidegger T, Lehotsky Á, et al. A large-scale assessment of hand hygiene quality and the effectiveness of the "WHO 6-steps." BMC Infect Dis 2013;13:249.

22. Longtin Y, Schneider A, Tschopp C, et al. Contamination of stethoscopes and physicians' hands after a physical examination. Mayo Clin Proc 2014;89:291-299.

23. Chadwick C. Infection control 4: good hand-hygiene practice for hospital patients. Nurs Times 2019;115:27-29.

24. Rational Use of Personal Protective Equipment for Coronavirus Disease (COVID-19): Interim Guidance, 27 February 2020. Geneva: WHO; 2020.

25. Babeluk R, Jutz S, Mertlitz S, Matiasek J, Klaus C. Hand hygieneevaluation of three disinfectant hand sanitizers in a community setting. PLoS One 2014;9:e111969.

26. Green LR, Radke V, Mason R, et al. Factors related to food worker hand hygiene practices. J Food Protect 2007;70:661-666.

27. Taylor JK, Basco R, Zaied A, Ward C. Hand hygiene knowledge of college students. Am Soc Clin Lab Sci 2010;23:89-93.

28. White C, Kolble R, Carlson R, Lipson N. The impact of a health campaign on hand hygiene and upper respiratory illness among college students living in residence halls. J Am Coll Health 2005;53:175-181.

29. Appiah-Brempong E, Harris MJ, Newton S, Gulis G. A framework for designing hand hygiene educational interventions in schools. Int J Pub Health 2018;63:251-259.

30. Willmott M, Nicholson A, Busse H, MacArthur GJ, Brookes S, Campbell R. Effectiveness of hand hygiene interventions in reducing illness absence among children in educational settings: a systematic review and metaanalysis. Arch Dis Child 2016;101:42-50. 
31. Gurses AP, Tschudy MM, McGrath-Morrow S, et al. Overcoming COVID-19: what can human factors and ergonomics offer? J Patient Saf Risk Manage 2020. doi.org/10.1177/2516043520917764.

32. Mumma JM, Durso FT, Casanova LM, et al. Variability in the duration and thoroughness of hand hygiene. Clin Infect Dis 2019;69:S221-S223.

33. Mumma JM, Durso FT, Ferguson AN, et al. Human factors risk analyses of a doffing protocol for Ebola-level personal protective equipment: mapping errors to contamination. Clin Infect Dis 2018;66:950-958.

34. Carayon P. Handbook of Human Factors and Ergonomics in Health Care and Patient Safety. Boca Raton, FL: CRC Press; 2016.
35. Gurses AP, Ozok AA, Pronovost PJ. Time to accelerate integration of human factors and ergonomics in patient safety. BMJ Qual Saf 2012;21: 347-351.

36. Pennathur PR, Thompson D, Abernathy III JH, et al. Technologies in the wild (TiW): human factors implications for patient safety in the cardiovascular operating room. Ergonomics 2013;56:205-219.

37. Urbaniak G, Plous S. Research randomizer [computer software]. https:// www.randomizer.org/. Published 2013. Accessed January 31, 2020.

38. Durso FT, Geldbach KM, Corballis P. Detecting confusion using facial electromyography. Human Factors 2012;54:60-69. 